\title{
Multi-Functional Diagnostic Method with Tracer-Encapsulated Pellet Injection
}

\author{
Shigeru SUDO ${ }^{1,3)}$, Naoki TAMURA ${ }^{1)}$, Diana KALININA ${ }^{1)}$, Igor VINYAR ${ }^{2)}$, Kuninori SATO ${ }^{1,3)}$, \\ Evgeny VESHCHEV ${ }^{3)}$ Pavel GONCHAROV ${ }^{1)}$, Tetsuo OZAKI ${ }^{1,3)}$, Shigeru INAGAKI ${ }^{1}$, \\ Hisamichi FUNABA $^{1)}$, Sadatsugu MUTOH ${ }^{1,3)}$, Yuri IGITKHANOV ${ }^{4}$, Liu YI ${ }^{5}$, Byron PETERSON ${ }^{1)}$ \\ Dan STUTMAN ${ }^{6)}$, Michael FINKENTHAL ${ }^{6)}$ and LHD Experimental Group ${ }^{1)}$ \\ ${ }^{1)}$ National Institute for Fusion Science, Toki 509-5292, Japan \\ 2) PELIN Laboratory, Ltd., 2, Admiral Makarov Str., Moscow, 125212, Russia \\ ${ }^{3)}$ Graduate University for Advaneced Studies, Hayama 240-0193, Japan \\ ${ }^{4)}$ Max-Planck-Institut für Plasmaphysik, EURATOM-Association, D-85748 Garching, Germany \\ ${ }^{5}$ Southwestern Institute of Physics, P.O.Box 432, Chengdu, Sichuan 610041, China \\ 6) Johns Hopkins University, Baltimore, MD 21218, USA
}

(Received 13 December 2006 / Accepted 1 May 2007)

\begin{abstract}
In order to obtain a better understanding of impurity transport in magnetically confined plasmas, a TracerEncapsulated Soild PELlet (TESPEL) has been developed. The essential points of the TESPEL are as follows: the TESPEL has a double-layered structure, and a tracer impurity, the amount of which can be known precisely, is embedded as an inner core. This structure enables us to deposit the tracer impurity locally inside the plasma. From experiences of developing the TESPEL production technique and its injection experiments, it became clear that various plasma properties can be studied by the TESPEL injection. There are not only impurity transport in the plasma but also transport both outside and inside of the magnetic island O-point, heat transport and high-energy neutral particle flux. Therefore, the TESPEL injection has a favorable multi-functional diagnostic capability. Furthermore a Tracer-Encapsulated Cryogenic PELlet (TECPEL) has been also developed. The TECPEL has an advantage over the TESPEL in terms of no existence of carbons in the outer layer. The TECPEL injector was installed at LHD in December 2005, and the preliminary injection experiments have been carried out.
\end{abstract}

(C) 2007 The Japan Society of Plasma Science and Nuclear Fusion Research

Keywords: pellet injection, tracer-encapsulated solid pellet, tracer-encapsulated cryogenic pellet, impurity transport, heat transport, magnetic island, pellet charge exchange

DOI: $10.1585 /$ pfr.2.S1013

\section{Introduction}

In recent years, steady progress has been made in the study of magnetically confined plasmas for establishing a cost-effective fusion reactor. However, the physical mechanism of the particle and heat transport in the plasma has not yet been fully clarified, especially regarding the impurity transport. The reason for the lack of understanding of the impurity transport is mainly due to the disadvantage of the conventional methods using impurity pellet injection [1] or laser blow-off technique [2]. These methods are difficult to measure the local properties, because of a broad source profile of the injected impurity and the unclarity of the total amount of the injected impurity. In order to overcome these problems and establish the local measurement of the impurity transport, a Tracer-Encapsulated Soild PELlet (TESPEL) $[3,4]$ has been developed. The TESPEL injection experiments have been performed diligently in the Large Helical Device (LHD), which has a heliotron type magnetic configuration [5]. Typical ex- perimental conditions are as follows; major radius at the magnetic axis, $R_{\mathrm{ax}}=3.6 \mathrm{~m}$, an averaged plasma minor radius $a_{p}=0.64 \mathrm{~m}$ (the resulting plasma volume is $30 \mathrm{~m}^{3}$ ), toroidal magnetic field strength $B_{T}=2.75 \mathrm{~T}$. From the experiences of developing the TESPEL production technique and its injection experiments, it became clear that various plasma properties can be studied with the TESPEL injection. These are not only impurity transport in the plasma, but also transport both outside and inside of the magnetic island O-point, heat transport and high-energy neutral particle flux. This is essentially owing to a flexibility of pellet size and a wide range of tracer material choice according to the experimental purpose. In this paper, experimental results, which are obtained with the TESPEL injection, are mainly reviewed, focusing on recent results. The TESPEL itself is briefly explained in Section 2. 

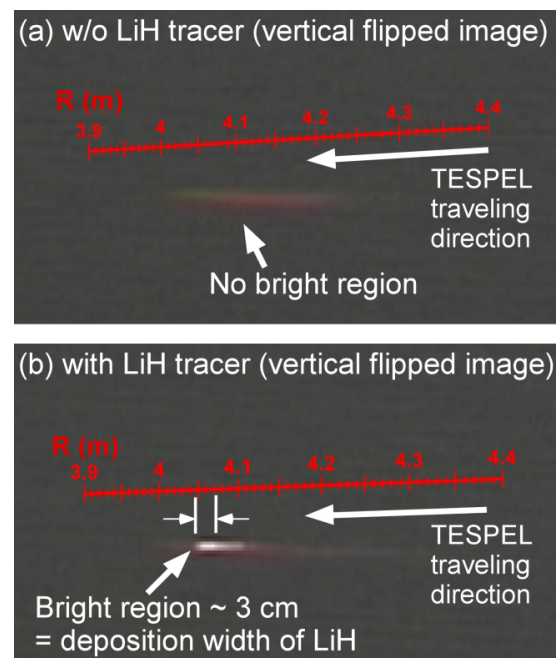

Fig. 1 CCD images of the ablating TESPEL through a Li I filter $(\lambda=671.3 \mathrm{~nm}, \delta \lambda=3.6 \mathrm{~nm})$ in the case (a) without $\mathrm{LiH}$ tracer particles and (b) with those (from figure 4 in [7]).

\section{Tracer-Encapsulated Solid Pellet (TESPEL)}

A TESPEL consists of polystyrene $\left(-\mathrm{CH}\left(\mathrm{C}_{6} \mathrm{H}_{5}\right) \mathrm{CH}_{2-}\right)$ polymer [6] as an outer shell, the diameter of which ranges from $400 \mu \mathrm{m}$ to $900 \mu \mathrm{m}$, and tracer particles as an inner core. Owing to its structure, the tracer particles encapsulated in the TESPEL can be deposited locally in the plasma, which can be confirmed by a CCD camera measurement as shown in Fig. 1 [7]. In the case of Fig. 1(b), the deposition width of a lithium hydride $(\mathrm{LiH})$ tracer is about $3 \mathrm{~cm}$, which is in good agreement with the result of another optical measurement. The deposited amount of the tracer impurity in the plasma can be known precisely during the TESPEL production procedure. One can choose a tracer material among a wide range of solid materials (available even in the form of powder).

\section{Impurity Transport Study with the TESPEL Injection}

Titanium (Ti) impurity transport in the plasma has been studied by measuring temporal evolutions of emission lines from the highly ionized Ti tracer impurity with a soft X-ray pulse height analyzer and a vacuum ultraviolet spectrometer, respectively [8]. In order to estimate transport coefficients, such as diffusion coefficient $D$, convective velocity $V$, a time-dependent MIST code [9] and a collisional-radiative atomic physics model have been used. When the electron density in the target plasma is increased from the value of $1.8 \times 10^{19} \mathrm{~m}^{-3}$ to that of $3.5 \times 10^{19} \mathrm{~m}^{-3}$, the $D$ decreases from $0.1 \mathrm{~m}^{2} / \mathrm{s}$ to $0.06 \mathrm{~m}^{2} / \mathrm{s}$ and an inward convection velocity arises $(0.8 \mathrm{~m} / \mathrm{s}$ at the plasma edge). This indicates that the $\mathrm{Ti}$ impurities are accumulated in the core plasma under the higher electron density. It is important to study how to avoid such an impurity accumulation in the core plasma in terms of preventing the occurrence of a radiative collapse. In order to explore the possibility of preventing the impurity accumulation by an additional heating, the effect of electron cyclotron heating $(\mathrm{ECH})$ on the impurity transport is also being studied.

So far, even for the stellarator plasmas, the impurity transport code originally developed for the tokamak configuration (e.g. MIST and STRAHL [10]), has been used to evaluate the transport coefficients. In order to improve this situation, a Heliotron-compatible impurity transport code STRAHL has been developed under the collaboration between National Institute for Fusion Science in Japan and Max-Planck Institut für Plasmaphysik in Germany. In order to convert the original STRAHL code into a Heliotroncompatible one, a neoclassical transport coefficients solver corresponding to LHD, an ambipolar radial electric field solver and LHD Magnetic configuration metrics are incorporated. Evaluation of impurity transport coefficients for signals obtained by the TESPEL experiments using the Heliotron-compatible STRAHL code is in progress now.

\section{Transport both Outside and Inside of a Magnetic Island O-point}

A magnetic island, which is formed due to distortion of magnetic surfaces, could affect the transport of heat and particle. The LHD experiment shows, indeed, the heat transport inside the magnetic island O-point is smaller by one order of magnitude than that outside the island $\mathrm{O}$ point [11]. Owing to the feature of the TESPEL, the tracer impurity can be deposited inside the magnetic island $\mathrm{O}$ point by the TESPEL injection [12]. Thus the impurity transport both outside and inside of the island O-point can be studied by observing a radiation from the ionized tracer. Under the present situation, a tomography image based on signals from absolute extreme ultraviolet photodiode (AXUVD) arrays viewed on the semi-tangential plane of LHD can provide us with information on the dynamic behavior of the tracer deposited in the island O-point. The tomography image in the case with the tracer deposited in the island O-point indicates that the radiation region, which corresponds to the tracer ablation position, diffuses more slowly compared to that in the case with the tracer deposited outside the island O-point. Thus this experimental result suggests that the magnetic island $\mathrm{O}$-point has a good confinement property for particles.

\section{Heat Transport Change due to the TESPEL Injection}

When the TESPEL is injected into the LHD plasmas, the edge region of the LHD plasmas is abruptly cooled and the resulting cold pulse (negative electron temperature perturbation) propagates from the edge to the core. A transient transport analysis on the cold pulse propagation in the plasma allows us to obtain transient properties of the 


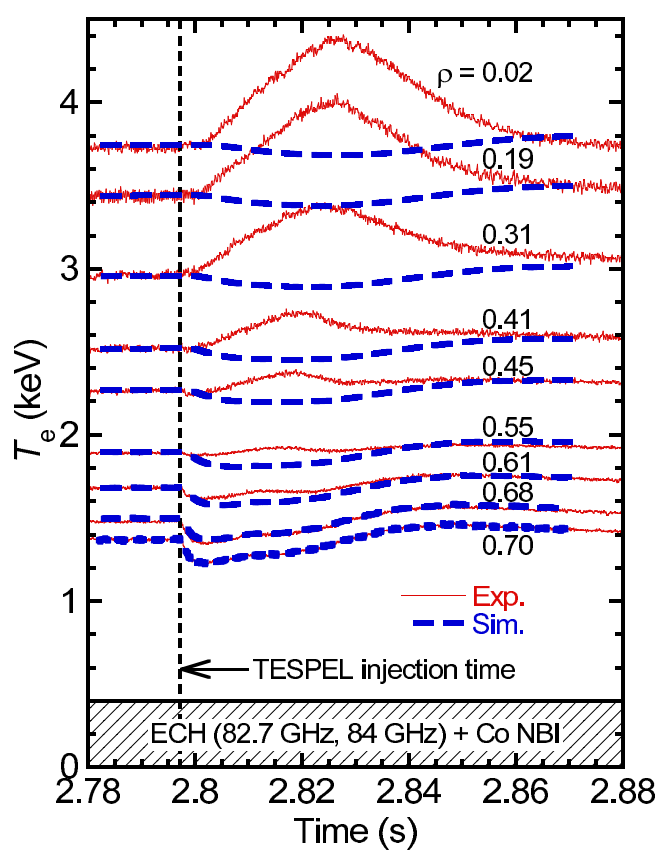

Fig. 2 Temporal evolution of electron temperature measured (solid lines) with the ECE radiometer at different normalized minor radii. The electron temperature simulated based on a simple diffusion model (broken lines) is also plotted. The TESPEL injection time is indicated as the vertical dashed line (from figure 1(c) in [14]).

heat transport [13]. The surprising result in the cold pulse experiment on LHD is that the core electron temperature is abruptly increased in response to the edge cooling induced by the TESPEL injection, as seen in Fig. 2 [14]. During the rising phase of the core electron temperature, no significant change in the low- $m$ magnetohydrodynamics modes is observed and no density peaking takes place. The transient transport analysis indicates the heat transport in the core plasma is abruptly improved without changing local parameters in the region of interest. Thus this phenomenon can be regarded as a so-called nonlocal electron temperature rise observed in many tokamaks (e.g. [15]). Although the physical mechanism, which determines the fast change of heat transport, remains to be clarified, the nonlocal electron temperature rise in response to the edge cooling on LHD clearly shows that the TESPEL injection is a powerful probe for the dynamics of the heat transport.

\section{High-Energy Neutral Particle Mea- surement with Pellet Charge Ex- change Method}

It is one of the advantages in Pellet Charge eXchange (PCX) diagnostic to obtain a radial information of the high energy particles. Since a neutral particle analyzer (NPA) for the measurement of charge exchange neutral particles is installed just behind the TESPEL trajectory as shown in Fig. 3 (a), the time trace of the NPA signal can be trans-
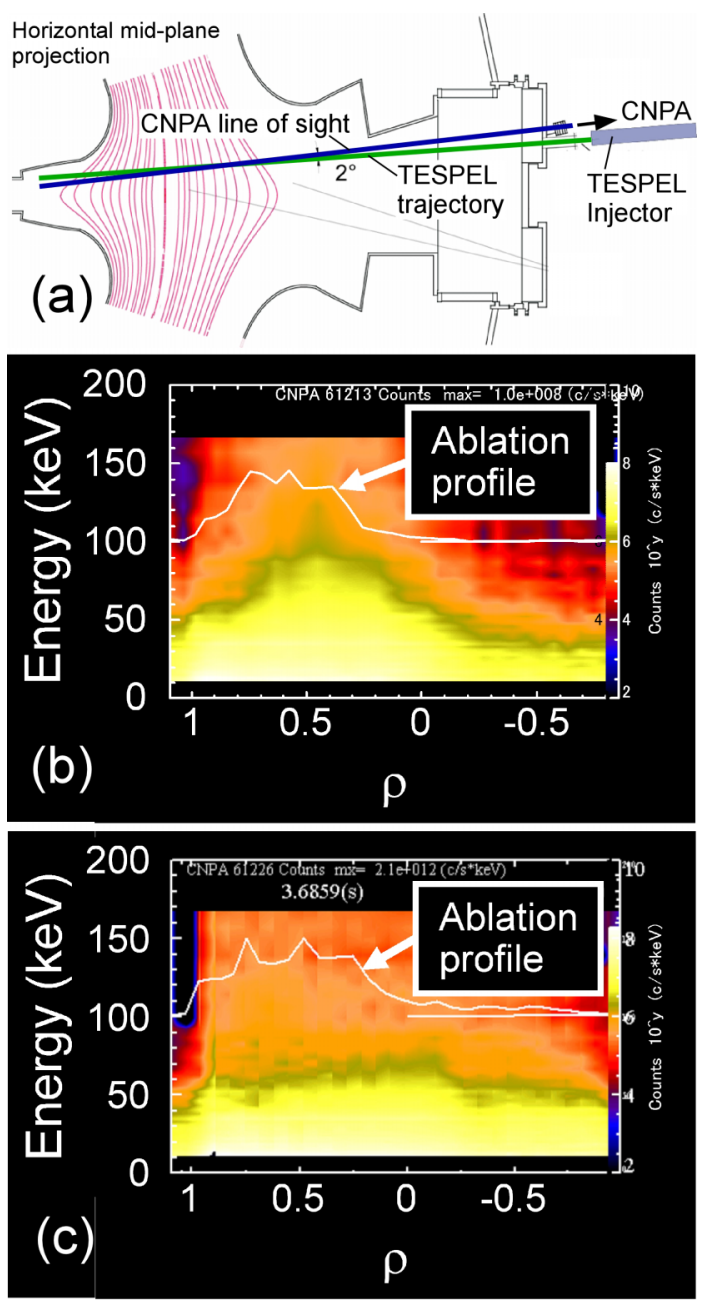

Fig. 3 (a) Schematic layout of PCX diagnostic on LHD. The contour plot for the high-energy particle flux measured with the CNPA in the case of (b) ICH off-axis heating mode (from figure 4 in [16]) and (c) ICH on-axis heating mode.

ferred to the radially-resolved information [16, 17]. The flux of the neutral particles entering the NPA is the multiplication of an emitted neutral flux from the TESPEL ablation cloud, a solid angle of viewing area and an attenuation factor through the plasma. Here the emitted neutral flux is the multiplication of a pellet cloud density, a fast ion distribution function and a neutralization factor. It is used for the NPA a compact neutral particle analyzer (CNPA, 40 channels, measurable energy range: $0.8 \sim$ $168 \mathrm{keV}$, energy resolution: $\sim$ several $\%$, and temporal resolution: $0.1 \mathrm{~ms}$ ), which is developed in the Ioffe Institute. It has a compact design of $30 \mathrm{~cm} \times 30 \mathrm{~cm}$ with a diamond like carbon film for ionization, a permanent magnet and rectangular shaped channneltrons. The typical TESPEL velocity $(500 \mathrm{~m} / \mathrm{s})$ brings the spatial resolution of $\sim 5 \mathrm{~cm}$ by the sampling time of $0.1 \mathrm{~ms}$. A typical PCX experiment has been done for plasmas heated by ion-cyclotron wave heating (ICH), the frequency of which is $38.47 \mathrm{MHz}$. In this experiment, a hydrogen minority heating is ex- 
pected. The plasmas are sustained not only the ICH wave but also the perpendicular neutral beam injection ( $\mathrm{p}-\mathrm{NBI})$. Just after the TESPEL injection, the plasma stored energy $W_{p}$ slightly decreases and the density increases. TESPEL reaches $\rho(=r / a)=0.1$ in this experiment, which means the radially-resolved information of the high-energy particles in the almost whole plasma can be obtained. As shown in Fig. 3(b), in the case of the ICH off-axis heating mode $\left(R_{\mathrm{ax}}=3.6 \mathrm{~m}, B_{T}=1.375 \mathrm{~T}\right)$, the high-energy particle flux measured is increased around $\rho=0.5$, where corresponds to the $\mathrm{ICH}$ resonance layer. The radial position of the $\mathrm{ICH}$ resonance layer can be varied by changing the magnetic field strength. When the magnetic field strength is changed from $B_{T}=1.375 \mathrm{~T}$ to $B_{T}=1.250 \mathrm{~T}$, the $\mathrm{ICH}$ resonance layer is on the magnetic axis (on-axis heating mode) and its location disappears from the TESPEL trajectory. Indeed, no significant increment of high-energy particle flux is observed with the CNPA, as shown in Fig. 3(c). These experimental results clearly show that the PCX diagnostic has a good capability of measuring the local high-energy particle flux in the plasma.

\section{Tracer-Encapsulated Cryogenic Pellet (TECPEL)}

The outer layer of TESPEL consists of polystyrene, that is, hydrogen and carbon. Since the carbon is an impurity for the bulk plasma, it should be eliminated for obtaining the better condition of diagnostics, such as charge exchange recombination spectroscopy. In order to get rid of the carbon from the tracer-encapsulated pellet, a TracerEncapsulated Cryogenic PELlet (TECPEL) has been developed [18]. The outer layer of the TECPEL consists of pure solid hydrogen. The TECPEL is formed in-situ in the gun barrel of the injector, and all the process can be monitored by a CCD camera with a suitable illumination and an optical system including a long-focus lens. Figure 4 shows snapshots of the in-flight TECPELs without and with a carbon tracer (carbon sphere with a $0.23 \mathrm{~mm}$ diameter). The integrity of TECPEL is confirmed from these photos, which can be taken for every pellet. The TECPEL injector was installed at LHD in December 2005, and the preliminary experiments of the TECPEL injection into the LHD plasmas have been successfully implemented.

\section{Summary}

As a result of implementing the TESPEL injection experiments on LHD, the TESPEL injection method promoted strongly the following important issues: (a) Estimation of the impurity transport properties, such as diffusion coefficient $D$ and inward pinch velocity $V$, (b) Understanding of the dynamics of the heat transport (the most interesting example is that core electron temperature rise

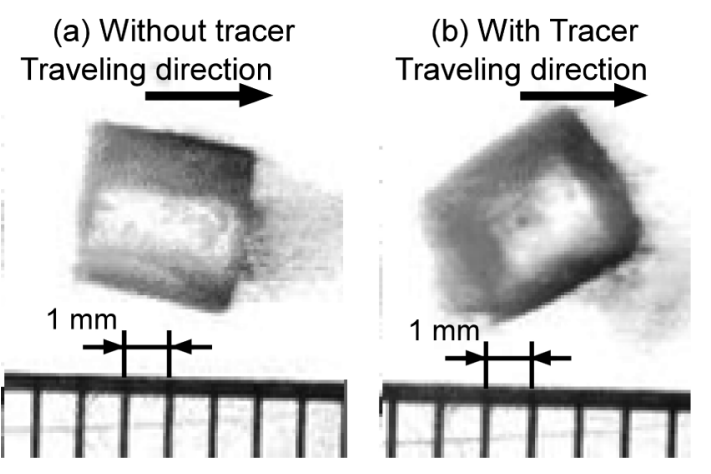

Fig. 4 Snapshots of the in-flight TECPELs (a) without and (b) with a carbon tracer (sphere-shaped with a $0.23 \mathrm{~mm}$ diameter) (from figures 8(c)-(d) in [18]).

invoked by the edge cooling due to the TESPEL injection), (c) Study of the transport properties near and in the magnetic island O-point, and (d) Study of the radially-resolved energy distribution of the high-energy particles with aid of pellet charge exchange (PCX) process. Furthermore, the more advanced TECPEL technique has been established, and the preliminary experiments of the TECPEL injection into the LHD plasmas have been done in December 2005. These experimental results show a good diagnostic capability of TESPEL/TECPEL for investigating the plasma properties. The authors acknowledge all of the technical staff of NIFS for their excellent support. This work is supported by a budgetary Grant-in-Aid No.NIFS06ULHH510 and a Grant-in-Aid for Scientific Research from Japan Society for the Promotion of Science No.15340201.

[1] K. Khlopenkov et al., Fusion Eng. Design 34-35, 337 (1997).

[2] H. Kaneko et al., Nucl. Fusion 27, 1075 (1987).

[3] S. Sudo et al., J. Plasma Fusion Res. 69, 1349 (1993).

[4] K. Khlopenkov et al., Rev. Sci. Instrum. 69, 3194 (1998).

[5] O. Motojima et al., Phys. Plasma 65, 1843 (1999).

[6] K. Nagai et al., J. Polymer. Sci., A: Polym. Chem. 38, 3412 (2000).

[7] N. Tamura et al., J. Plasma Fusion Res. SERIES, 4, 442 (2001).

[8] N. Tamura et al., Plasma Phys. Control. Fusion 45, 27 (2003).

[9] R.A. Hulse, Nucl. Technol/Fusion, 3, 259 (1983).

[10] K. Behringer, JET Report No. JET-R87, 08 (1987).

[11] S. Inagaki et al., Phys. Rev. Lett. 92, 055002-1 (2004).

[12] N. Tamura et al., J. Plasma Fusion Res. 78, 837 (2002).

[13] S. Inagaki et al., Nucl. Fusion 46, 133 (2006).

[14] N. Tamura et al., Phys. Plasmas 12, 110705 (2005).

[15] K.W. Gentle et al., Phys. Rev. Lett. 74, 3620 (1995).

[16] T. Ozaki et al., Rev. Sci. Instrum. 77, 10E917-1 (2006).

[17] P.R. Goncharov et al., Rev. Sci. Instrum. 77, 10F119-1 (2006).

[18] S. Sudo et al., Rev. Sci. Instrum. 76, 053507 (2005). 